Pacific Journal of Mathematics

COUNTEREXAMPLES TO SOME CONJECTURES ABOUT 


\title{
COUNTER-EXAMPLES TO SOME CONJECTURES ABOUT DOUBLY STOCHASTIC MEASURES
}

\author{
V. LOSERT
}

\begin{abstract}
Some new types of doubly stochastic measures are constructed. Using measure preserving transformations, one can construct examples of nontrivial extreme doubly stochastic measures which are absolutely continuous with respect to another extreme doubly stochastic measure (disproving a conjecture by Feldman). By combinatorial arguments, one gets an extreme doubly stochastic measure that is not concentrated on a countable union of function graphs and whose support is the whole unit square.
\end{abstract}

o. Let $I$ be the unit interval, $m$ the ordinary Lebesgue measure on $I$. A probability measure $\mu$ on $I \times I$ is called doubly stochastic, if its marginal distributions coincide with $m$ (i.e., $\mu(A \times I)=\mu(I \times A)=$ $m(A)$ for each Borel set $A \subseteq I)$. This is thought of as a continuous analogue of the notion of a doubly stochastic matrix (see [8] for a survey of results about doubly stochastic matrices). By a theorem of G. Birkhoff and von Neumann, the extreme points of the set of doubly stochastic matrices are the permutation matrices. The continuous analogue of a permutation matrix would be the graph of a bijective, measure preserving function, but it is easy to construct extreme points that are not of this type (a deep study, showing that for some purposes these special measures may well suffice has been given in [13]). On the other hand, all concrete examples of extreme doubly stochastic measures (e.d.s.m.) that can be found in the literature are concentrated on the graphs (or inverse graphs) of functions, mostly even linear functions and there was some common belief that any double stochastic measure must in some sense be made up from graphs (cf. the beginning of $\S 2$ of [2]). A functional analytic characterization of the e.d.s.m. has been given by Douglas [4] and Lindenstrauss [7]. Several authors have tried to generalize properties of permutation matrices to these measures, see e.g., [1], [2], [10]. One of the aims of this paper is to present some new constructions of e.d.s.m. which will also disprove some natural conjectures. The second construction yields a measure that is not concentrated on graphs. While the measures in the first construction are still concentrated on two graphs, it turns out that even in this case the geometric interrelations are much more complicated. In [2] the following conjecture (attributed to J. Feldman) was mentioned: if $\mu$ is an e.d.s.m. and if $\nu$ is a doubly stochastic measure which is 
absolutely continuous with respect to $\mu$, then $\nu=\mu$. In [2] this conjecture was confirmed by Brown and Shiflett for a class of extreme doubly stochastic measures which is geometrically related to permutation matrices. In Theorem 1 of our paper we will give a functional analytic characterization of measures $\mu$ that satisfy a slightly stronger property as above ( $\nu$ need not be positive - the result of [2] holds also for this stronger property). It is along the lines of the results by Douglas in [5]. Then we study a special class of doubly stochastic measures, defined in a certain way by a measure preserving transformation $T$. A similar type of measures (but with a different behavior) has been studied in [12]. In Theorem 2 we give conditions on $T$ that ensure that the corresponding measure be extremal (resp. satisfies the properties of Theorem 1). Then it is easy to give examples of transformations for which the properties of Theorem 1 do not hold and which disprove also Feldman's conjecture mentioned above. The idea to use ergodicity properties of transformations for the construction of doubly stochastic measures was first used in [13] p. 87.

The second part of the paper concerns the support of an e.d.s.m. It has been proved in [7] that any such measure is singular with respect to the ordinary Lebesgue measure $m \otimes m$ on the unit square, i.e., it is concentrated on a set of $m \otimes m$ - measure zero. Nevertheless, we will give an example showing that the support of the measure may be the whole unit square, i.e., the measure is not concentrated on a closed set whose $m \otimes m$-measure is less than one. This measure has also the property that any graph (or inverse graph) of some measurable function has measure zero (Theorem 3). I would like to thank S. Graf for bringing these problems to my attention and also for several references to the literature.

1. If $m^{\prime}$ is an arbitrary probability measure on $I=[0,1]$, we write $E_{m}$, for the set of all probability measures on $I \times I$ whose marginal distributions are equal to $m^{\prime}$. We write $\mu^{\prime} \prec \mu$, if $\mu^{\prime}$ is absolutely continuous with respect to $\mu$.

THEOREM 1. If $\mu \in E_{m}$, then the following statements are equivalent:

(i) If $\nu$ is an arbitrary (complex) measure which is absolutely continuous with respect to $\mu$ and whose marginal distributions are equal to $m$, then $\nu=\mu$.

(ii) The space of functions $F=\left\{(x, y) \rightarrow f(x)+g(y): f, g \in L^{\infty}(m)\right\}$ is weak *-dense in $L^{\infty}(\mu)$.

(iii) If $m^{\prime}$ is a probability measure which is absolutely continuous with respect to $m$ and $\mu^{\prime} \in E_{m}$, is absolutely continuous with 
respect to $\mu$, then $\mu^{\prime}$ is an extreme point in $E_{m^{\prime}}$ (i.e., $\mu$ is a sort of "hereditary extreme point").

The main content of the theorem may be rephrased as follows: if $F$ is $w^{*}$-dense in $L^{\infty}(\mu), m^{\prime} \prec m, \mu^{\prime} \prec \mu$, and $\mu^{\prime} \in E_{m^{\prime}}$, then $F$ is also $w^{*}$-dense in $L^{\infty}\left(\mu^{\prime}\right)$.

Proof. (ii) $\Rightarrow$ (i) is Theorem 4 of [5].

(i) $\Rightarrow$ (ii) follows also from the methods of [5]: if $u \in L^{1}(\mu)$ annihilates the space $F$, then $\nu=(1+u) \mu$ has the same marginal distributions as $\mu$.

(iii) $\Rightarrow$ (ii). Given finitely many elements $u_{1}, \cdots, u_{k} \in L^{1}(\mu), \varepsilon>0$ and $h \in L^{\infty}(\mu)$, there exists a nonnegative function $u \in L^{1}(\mu)$ and $c>0$ such that $u(x, y)=u(y, x)$ for all $x, y \in I, \int u(t) d \mu(t)=1$ and $\left|u_{i}\right| \leqq c u$ for $i=1, \cdots, k$. Put $\mu^{\prime} \doteq u \mu$ and $m^{\prime}=\left(\int u(\cdot, y) d m(y)\right) m$; then $m^{\prime} \prec m$ and $\mu^{\prime} \in E_{m^{\prime}}$. By (iii) and Theorem 1 of [4], there exists a function $h_{0} \in F$ such that $\left\|h-h_{0}\right\|_{L^{1}\left(\mu^{\prime}\right)}<\varepsilon / c$. This implies clearly $\left|\int u_{i}(t)\left(h(t)-h_{0}(t)\right) d \mu(t)\right|<\varepsilon$ for $i=1, \cdots, k$.

(ii) $\Rightarrow$ (iii). By Theorem 1 of [4] it is sufficient to show that $F$ is dense in $L^{\infty}(\mu)$ with respect to the norm topology induced by $L^{1}\left(\mu^{\prime}\right)$.

The result follows from a general lemma on convex subsets of $L^{\infty}(\mu)$.

LEMma. If $F$ is a convex subset of $L^{\infty}(\mu)$ and $\mu^{\prime} \prec \mu$, then the $w^{*}$-closure of $F$ in $L^{\infty}(\mu)$ is contained in the closure taken with respect to the norm topology induced by $L^{1}\left(\mu^{\prime}\right)$.

Proof. Since $\mu^{\prime}<\mu$, we get $\mu^{\prime}=u \mu$ for some $u \in L^{1}(\mu)$. The continuous functionals for the $w^{*}$-topology are given by elements of $L^{1}(\mu)$. The continuous functionals for the norm topology induced by $L^{1}\left(\mu^{\prime}\right)$ are represented by elements of $\left\{h u: h \in L^{\infty}(\mu)\right\} \subseteq L^{1}(\mu)$ (if one uses the same duality as above). Now the lemma follows easily from the Hahn Banach theorem ([11], Ch. II, 9.2).

Now let $T:[0,1 / 2] \rightarrow[0,1 / 2]$ be an arbitrary measure preserving transformation. We consider measures $\mu$ supported by the following four sets: $F_{1}=\{(x, T x) ; 0 \leqq x \leqq 1 / 2\}, F_{2}=\{(x, x-1 / 2) ; 1 / 2 \leqq x \leqq 1\}$, $F_{3}=\{(x, x+1 / 2) ; 0 \leqq x \leqq 1 / 2\}, F_{4}=\{(x, x): 1 / 2 \leqq x \leqq 1\}$ (i.e., the unit square is partitioned into four congruent sub squares. In the first of them we consider the graph of $T$, in the other squares, the diagonal). Let $j:[0,1 / 2] \rightarrow F_{1}$ be defined by $j(x)=(x, T x)$. $j$ induces a bijective correspondence between measures on $[0,1 / 2]$ and $F_{1}$. 
The corresponding measures will be denoted by the same letter. If $\mu$ is supported by $F=F_{1} \cup F_{2} \cup F_{3} \cup F_{4}$, then $\mu_{1}$ shall denote its restriction to $F_{1}$.

THEOREM 2. If $\mu$ is a doubly stochastic measure, supported by $F$, then the following statements hold:

(i) $\mu$ is uniquely determined by $\mu_{1}=\mu \mid F_{1}$. Conversely, $a$ measure $\mu_{1}$ on $[0,1 / 2]$ induces a doubly stochastic measure on $F$, iff $\mu_{1}$ is $T$-invariant and $0 \leqq \mu_{1} \leqq m$.

(ii) Write $\mu_{1}=h_{1} m\left(h_{1} \in L^{\infty}(m), 0 \leqq h_{1} \leqq 1\right)$. $\mu$ is an extreme doubly stochastic measure iff $\mu_{1}$ (resp. $h_{1}$ ) has the following property: if $A \subseteq[0,1 / 2]$ is a T-invariant, measurable set with $\mu_{1}(A)>0$, then ess $\sup _{x \in A} h_{1}(x)=1$.

(iii) $\mu$ satisfies the conditions of Theorem 1 , iff $\mu_{1}$ (resp. $h_{1}$ ) has the following property: if $A \subseteq[0,1 / 2]$ is a T-invariant, measurable set with $\mu_{1}(A)>0$, then $m\left\{x \in A: h_{1}(x)=1\right\}>0$. In particular, if $\left\{x: h_{1}(x)=1\right\}$ has m-measure zero, then $\mu$ does not satisfy the conditions of Theorem 1.

Proof. Assume that $\mu$ is given. Let $A$ be a measurable subset of $[0,1 / 2]$.

Since $\mu$ is doubly stochastic, we get:

$$
\begin{aligned}
m(A)=\mu(I \times A) & =\mu\left((I \times A) \cap F_{1}\right)+\mu\left((I \times A) \cap F_{2}\right) \\
& =\mu\left(T^{-1} A \times A\right)+\mu([1 / 2,1] \times A) \\
& =\mu_{1}\left(T^{-1} A\right)+\mu((A+1 / 2) \times[0,1 / 2]) .
\end{aligned}
$$

Similarly

$$
\begin{aligned}
m(A) & =\mu((A+1 / 2) \times I)=\mu((A+1 / 2) \times[0,1 / 2])+\mu((A+1 / 2) \times[1 / 2,1]) \\
& \underset{(1)}{=} m(A)-\mu_{1}\left(T^{-1} A\right)+\mu([1 / 2,1] \times(A+1 / 2)) .
\end{aligned}
$$

This gives:

$$
\mu([1 / 2,1] \times(A+1 / 2))=\mu_{1}\left(T^{-1} A\right) .
$$

Furthermore

$$
\begin{aligned}
m(A)=\mu(I \times(A+1 / 2))=\mu([0,1 / 2] \times(A+1 / 2))+ & \mu([1 / 2,1] \\
& \times(A+1 / 2)
\end{aligned}
$$

gives

$$
\mu([0,1 / 2] \times(A+1 / 2))=m(\Lambda)-\mu_{1}\left(T^{-1} A\right) .
$$

And finally 


$$
\begin{aligned}
m(A)=\mu(A \times I) & =\mu(A \times[0,1 / 2])+\mu(A \times[1 / 2,1]) \\
& \underset{(3)}{=} \mu_{1}(A)+m(A)-\mu_{1}\left(T^{-1} A\right) .
\end{aligned}
$$

The last equation shows that $\mu_{1}(A)=\mu_{1}\left(T^{-1} A\right)$, i.e., $\mu_{1}$ is $T$-invariant. (1), (2), (3) show that the values of $\mu$ on $F_{2}, F_{3}, F_{4}$ are uniquely determined by $\mu_{1}$. $\quad \mu_{1} \leqq m$ holds by (3).

Conversely, if one defines the measure $\mu$ on $F_{2}, F_{3}, F_{4}$ by the formulas (1), (2), (3), it is easily seen that $\mu$ is a doubly stochastic measure. This proves (i).

(ii). Assume that $A \subseteq[0,1 / 2]$ is $T$-invariant $\mu_{1}(A)>0,0<\alpha \leqq$ $1 / 2$ and $h_{1} \leqq 1-\alpha$ a.e. on $A$. Let $c_{A}$ be the characteristic function of $A$. If we put $\mu_{1}^{\prime}=\left(1+\alpha(1-\alpha)^{-1} c_{A}\right) \mu_{1}$ and $\mu_{1}^{\prime \prime}=\left(1-\alpha(1-\alpha)^{-1} c_{A}\right) \mu_{1}$, then it follows easily from (i) that $\mu_{1}^{\prime}$ and $\mu_{1}^{\prime \prime}$ define doubly stochastic measures $\mu^{\prime}$ and $\mu^{\prime \prime}$ such that $\mu=\left(\mu^{\prime}+\mu^{\prime \prime}\right) / 2$.

For the converse assume that $\mu=\left(\mu^{\prime}+\mu^{\prime \prime}\right) / 2$ and $\mu^{\prime} \neq \mu^{\prime \prime}$. Then we get measures $\mu_{1}^{\prime}$ and $\mu_{1}^{\prime \prime}$ such that $\mu_{1}=\left(\mu_{1}^{\prime}+\mu_{1}^{\prime \prime}\right) / 2$. Put $\mu_{1}^{\prime}=f^{\prime} \mu_{1}$ and $\mu_{1}^{\prime \prime}=f^{\prime \prime} \mu_{1}$. Then $f^{\prime}+f^{\prime \prime}=2$ and $f^{\prime} \neq f^{\prime \prime}$. Therefore we may assume that there exists $\beta>1$ such that $A=\left\{x: f^{\prime}(x)>\beta\right\}$ satisfies $\mu_{1}(A)>0$. Since $\mu_{1}$ and $\mu_{1}^{\prime}$ are $T$-invariant, the same holds for $A$. Since $\mu_{1}^{\prime}=f^{\prime} h m \leqq m$ by (i), we have $f^{\prime} h \leqq 1$ and therefore $h \leqq \beta^{-1}$ on $A$.

(iii). By an argument similar to (i), it can be shown that an arbitrary (complex) measure $\mu^{\prime}$ on $F_{1} \cup F_{2} \cup F_{3} \cup F_{4}$ with marginal distributions $m$ is uniquely determined by its restriction $\mu_{1}^{\prime}$ to $F_{1}$. $\mu_{1}^{\prime}$ has to be $T$-invariant furthermore $\mu^{\prime} \prec \mu$ if $\mu_{1}^{\prime} \prec \mu_{1}$ and $m-\mu_{1}^{\prime}<$ $m-\mu_{1}$ Now the same construction as in (ii) gives the result.

Example. It is now easy to give examples of extreme doubly stochastic measures which do not satisfy the properties of Theorem 1. Let $T_{0}: I \rightarrow I$ be an arbitrary ergodic transformation (with respect to $m)$, e.g., $T_{0}(x)=x+\alpha(\bmod 1), \alpha$ irrational. We consider the mapping $g: I \rightarrow[0,1 / 2]$ defined by $g(x)=x^{1 / 2} / 2$. Then $T=g \circ T_{0} \circ g^{-1}$ is an ergodic transformation of $[0,1 / 2]$ with invariant measure $g(m)=8 y m$. Now define $h_{1}(y)=2 y$ for $y \in[0,1 / 2]$. By Theorem $2, \mu_{1}=h_{1} m$ defines a doubly stochastic measure $\mu$, which is extremal (by (ii) - observe that $A=[0,1 / 2]$ is the only invariant set) but does not satisfy the properties of Theorem 1 (by (iii)). It is easily seen that there exists even a probability measure $\mu^{\prime} \neq \mu$ which is doubly stochastic and absolutely continuous with respect to $\mu$ (e.g., the diagonal measure on $\left.F_{2} \cup F_{3}\right)$.

2. In the next section we present another method to construct extreme doubly stochastic measures. It uses approximations by 
measures on finite subalgebras. Let $\Sigma$ be the $\sigma$-algebra of Borel sets on $I$. We will consider increasing sequences $\left(\Sigma_{n}\right)$ resp. $\left(\Sigma_{n}^{\prime}\right)$ of finite subalgebras of $I$, where $\Sigma_{n}\left(\Sigma_{n}^{\prime}\right)$ is generated by a partition $P_{n}\left(Q_{n}\right)$ of $I$ into finitely many subintervals. We will define a measure $\mu$ successively on the subalgebras $\Sigma_{n} \otimes \Sigma_{n}^{\prime}$. To assure that the limit measure is extremal, we will use an idea similar to [2] Thm. 1 and [3] Thm. 2. There is a connection between extremality and the existence of "loops". If $\mu$ is not extremal, one will also get such loops in one of the approximating algebras $\Sigma_{n} \otimes \Sigma_{n}^{\prime}$. The aim of the following construction will be, to cut off each such loop at a later step.

THEOREM 3. There exists an extreme doubly stochastic measure $\mu$ whose support is the whole space. In addition, $\mu$ has the following property: if $\mu=\int \mu_{1, x} d m(x)=\int \mu_{2, x} d m(x)$ are the disintegrations of $\mu$ with respect to the two coordinate projections ( $\mu_{1, x}$ is concentrated on $\{x\} \times I, \mu_{2, x}$ on $\left.I \times\{x\}\right)$, then the measures $\mu_{1, x}$ and $\mu_{2, x}$ are continuous a.e. (i.e., each point has measure zero). In particular any graph or inverse graph of some measurable function $f: I \rightarrow I$ has u-measure zero.

Proof. By induction we will define partitions $P_{n}=\left\{I_{\alpha}^{(n)}\right\}_{\alpha \in A^{(n)}}$ and $Q_{n}=\left\{J_{\alpha}^{(n)}\right\}_{\alpha \in A^{(n)}}$ of $I$. For $n=0$ we put $A^{(0)}=\{1\}, I_{1}^{(0)}=J_{1}^{(0)}=I$, $\mu(I \times I)=1$. Now assume that $P_{n}, Q_{n}$ have already been defined, and $\mu\left(I_{\alpha}^{(n)} \times J_{\beta}^{(n)}\right)>0$ for all $\alpha, \beta \in A^{(n)}$. We consider two different cases:

(a) if $n$ is even, we choose $k>\max \left\{(n+1) \mu\left(I_{\alpha}^{(n)} \times J_{\beta}^{(n)}\right)\left(m\left(I_{\alpha}^{(n)}\right)^{-1}+\right.\right.$ $\left.\left.m\left(J_{\beta}^{(n)}\right)^{-1}\right): \alpha, \beta \in A^{(n)}\right\}$. We put $A^{(n+1)}=A^{(n)} \times\{1, \cdots, k\}$. Each interval $I_{\alpha}^{(n)}$ is partitioned into $k$-subintervals $I_{\alpha, 1}^{(n+1)}, \cdots, I_{\alpha, k}^{(n+1)}$ of equal length. We put $P_{n+1}=\left\{I_{\alpha, j}^{(n+1)}: \alpha \in A^{(n)}, j=1, \cdots, k\right\} . \quad Q_{n+1}$ is constructed in the same way from $Q_{n}$. Finally $\mu\left(I_{\alpha, i}^{(n+1)} \times J_{\beta, j}^{(n+1)}\right)=k^{-2} \mu\left(I_{\alpha}^{(n)} \times J_{\beta}^{(n)}\right)$. Then it is easily seen that

$$
\begin{array}{r}
\mu\left(J_{\alpha}^{(n+1)} \times J_{\beta}^{(n+1)}\right)<(n+1)^{-1} \min \left(m\left(I_{\alpha}^{(n+1)}\right), m\left(J_{\beta}^{(n+1)}\right)\right) \\
\text { for } \alpha, \beta \in A^{(n+1)}, n+1 \text { odd } .
\end{array}
$$

(b) if $n$ is odd, let $r=r(n)$ be the cardinality of $A^{(n)} \times A^{(n)}$ (i.e., the number of atoms in $\Sigma_{n} \otimes \Sigma_{n}^{\prime}$ ). We choose $k>2^{n} r$ and put $A^{(n+1)}=A^{(n)} \times\{1, \cdots, k\}^{r}$. Let $\varphi_{n}: A^{(n)} \times A^{(n)} \rightarrow\{1, \cdots, r\}$ be a fixed bijection. Finally put $c_{n}=\min \left\{\mu\left(I_{\alpha}^{(n)} \times J_{\beta}^{(n)}\right): \alpha, \beta \in A^{(n)}\right\}$. To get $P_{n+1}$ (resp. $Q_{n+1}$ ) we partition each interval $I_{\alpha}^{(n)}$ (resp. $J_{\alpha}^{(n)}$ ) into $k^{r}$ subintervals $I_{\alpha i_{1} \cdots i_{r}}^{(n+1)}\left(\right.$ resp. $J_{\alpha i_{1} \cdots i_{r}}^{(n+1)}$ ) where $1 \leqq i_{1} \leqq k$ for $1=1, \cdots, r$, whose length will be decided later on. We put 


$$
\begin{array}{r}
\mu\left(I_{\alpha i_{1} \cdots i_{r}}^{(n+1)} \times J_{\beta i_{1} \cdots i_{s}+1 \cdots i_{r}}^{(n+1)}\right)=\left(\mu\left(I_{\alpha}^{(x)} \times J_{\beta}^{(n)}\right)-c_{n} k^{-2 r} r^{-2}\right) k^{1-r}(k-1)^{-1} \\
+c_{n} k^{-4 r} r^{-2} \text { if } s=\varphi_{n}(\alpha, \beta)
\end{array}
$$

and

$$
\mu\left(I_{\alpha i_{1} \cdots i_{r}}^{(n+1)} \times J_{\beta j_{1} \cdots j_{r}}^{(n+1)}\right)=c_{n} k^{-4 r} r^{-2}
$$

for all other choices of the indices $\left(1 \leqq i_{l}, j_{l} \leqq k, l=1, \cdots, r, \alpha, \beta \in\right.$ $\left.A^{(n)}\right)$. It is easily seen that the sum of the measures of all subrectangles of $I_{\alpha}^{(x)} \times J_{\beta}^{(n)}$ equals $\mu\left(I_{\alpha}^{(n)} \times J_{\beta}^{(n)}\right)$. The length of the interval $I_{\alpha}^{(n+1)}\left(\alpha \in A^{(n+1)}\right)$ is now determined by $m\left(I_{\alpha}^{(n+1)}\right)=\sum_{\beta \in A(n+1)} \mu\left(I_{\alpha}^{(n+1)} \times\right.$ $\left.J_{\beta}^{(n+1)}\right)$, similarly for $J_{\beta}^{(n+1)}$.

If $1 \leqq i_{1}<k$ for $l=1, \cdots, r$ and $\alpha, \beta \in A^{(n)}$, then we have by (5), (6):

$$
\begin{aligned}
\mu\left(I_{\alpha, i_{1} \cdots i_{r}}^{(n+1)} \times J_{\beta}^{(n)}\right)-k^{1-r}(k-1)^{-1} \mu\left(I_{\alpha}^{(n)} \times J_{\beta}^{(n)}\right) & =-c_{n} k^{-3 r} r^{-2}(k-1)^{-1} \\
& \leqq 0
\end{aligned}
$$

Summation over $\beta$ gives

$$
m\left(I_{\alpha, i_{1} \cdots i_{r}}^{(n+1)}\right)-k^{1-r}(k-1)^{-1} m\left(I_{\alpha}^{(n)}\right)=-c_{n} k^{-3 r} r^{-3 / 2}(k-1)^{-1} .
$$

Since by (4) $m\left(I_{\alpha}^{(n)}\right) \geqq n \mu\left(I_{\alpha}^{(n)} \times J_{\beta}^{(n)}\right)$ and $c_{n} \leqq \mu\left(I_{\alpha}^{(n)} \times J_{\beta}^{(n)}\right)$, we get:

$$
\begin{aligned}
m\left(I_{\alpha i_{1} \cdots i_{r}}^{(n+1)}\right) & \geqq \mu\left(I_{\alpha}^{(n)} \times J_{\beta}^{(n)}\right)\left(n k^{1-r}(k-1)^{-1}-k^{-3 r} r^{-3 / 2}(k-1)^{-1}\right) \\
& \geqq \mu\left(I_{\alpha}^{(n)} \times J_{\beta}^{(n)}\right)(n-1) k^{1-r}(k-1)^{-1} .
\end{aligned}
$$

Combined with (7), we find that: $m\left(I_{\alpha i_{1} \cdots i_{r}}^{(n+1)}\right) \geqq(n-1) \mu\left(I_{\alpha i_{1} \cdots i_{r}}^{(n+1)} \times J_{\beta}^{(n)}\right) \geqq$ $(n-1) \mu\left(I_{\alpha i_{1} \cdots i_{r}}^{(n+1)} \times J_{\beta j_{1} \cdots j_{r}}^{(n+1)}\right)$ for all $1 \leqq j_{l} \leqq k, l=1, \cdots, r$. Now put $I^{(n)}=\bigcup\left\{I_{\alpha i_{1} \cdots i_{r}}^{(n+1)}: \alpha \in A^{(n)}, i_{1}=k\right.$ for at least one $\left.l\right)$. Then

$$
m\left(I_{\alpha}^{(n+1)} \times J_{\beta}^{(n+1)}\right) \leqq(n-1)^{-1} m\left(I_{\alpha}^{(n+1)}\right) \text { for all } \beta \in A^{(n+1)},
$$

if $I_{\alpha}^{(n+1)}$ is not contained in $I^{(n)}$. If $\alpha \in A^{(n)}$ is fixed, then $I_{\alpha}^{(n)} \backslash I^{(n)}$ is a union of $(k-1)^{r}$ intervals for which (8) holds. Therefore

$$
\begin{aligned}
m\left(I_{\alpha}^{(n)} \backslash I^{(n)}\right) \geqq\left(1-k^{-1}\right)^{r-1} m\left(I_{\alpha}^{(n)}\right)\left(1-k^{-2 r-1} r^{-3 / 2}\right) & \geqq\left(1-2^{-n}\right)^{2} m\left(I_{\alpha}^{(n)}\right) \\
& \geqq\left(1-2^{-n+1}\right) m\left(I_{\alpha}^{(n)}\right)
\end{aligned}
$$

(by our choice of $k$ and since clearly $r \geqq 2^{n}$ ). Summing over $\alpha \in$ $A^{(n)}$, we get:

$$
m\left(I^{(n)}\right) \leqq 2^{1-n} .
$$

In the same way, we get an exceptional set $J^{(n)}$ with $m\left(J^{(n)}\right)<2^{1-n}$, such that $\mu\left(I_{\alpha}^{(n+1)} \times J_{\beta}^{(n+1)}\right)<(n-1)^{-1} m\left(J_{\beta}^{(n+1)}\right)$ for all rectangles not contained in $I \times J^{(n)}$.

Having defined all partitions $P_{n}, Q_{n}$, we want to extend $\mu$ to a measure on $\Sigma \otimes \Sigma$. This is done as follows: Let $\mu^{(n)}$ be the pro- 
bability measure on $I \times I$, whose restriction to each rectangle $I_{\alpha}^{(n)} \times$ $J_{\beta}^{(n)}\left(\alpha, \beta \in A^{(n)}\right)$ is a multiple of the ordinary Lebesgue measure and such that $\mu^{(n)}\left(I_{\alpha}^{(n)} \times J_{\beta}^{(n)}\right)=\mu\left(I_{\alpha}^{(n)} \times J_{\beta}^{(n)}\right)$. Let $\mu^{\prime}$ be a cluster point of the sequence $\left(\mu^{(n)}\right)$ in the weak topology of the set of all Radon measures on $I \times I$ with respect to continuous functions). Since each $\mu^{(n)}$ is doubly stochastic, the same is true for $\mu^{\prime}$. In particular, the boundary of each rectangle $I_{\alpha}^{(n)} \times J_{\beta}^{(n)}$ has $\mu^{\prime}$-measure zero. If $\left(\mu^{\left(n_{i}\right)}\right)$ is a subsequence of $\left(\mu^{(n)}\right)$ converging towards $\mu^{\prime}$, it follows from [9] Ch. II, Thm. 6.1 that $\mu^{\left(n_{i}\right)}\left(I_{\alpha}^{(n)} \times J_{\beta}^{(n)}\right)$ converges to $\mu^{\prime}\left(I_{\alpha}^{(n)} \times J_{\beta}^{(n)}\right)$. This shows that $\mu^{\prime}\left(I_{\alpha}^{(n)} \times J_{\beta}^{(n)}\right)=\mu\left(I_{\alpha}^{(n)} \times J_{\beta}^{(n)}\right)$, i.e., $\mu^{\prime}$ is an extension of $\mu$. (From now on we will again write $\mu$ for $\mu^{\prime}$.) We claim that $\mu$ has the properties stated in Theorem 3.

We have already remarked that $\mu$ is doubly stochastic. Let $\mu=$ $\int \mu_{1, x} d m(x)$ be the disintegration of $\mu$ with respect to the first coodinate projection $\left(\mu_{1, x}\right.$ is concentrated on $\left.\{x\} \times I\right)$. Similarly $\mu^{(n)}=$ $\int \mu_{1, x}^{(n)} d m(x)$. If $x \in I_{\alpha}^{(n)}\left(\alpha \in A^{(n)}\right)$ and $M$ is a Borel subset of $I$, then $\mu_{1, x}^{(n)}(M)=\mu^{(n)}\left(I_{\alpha}^{(n)} \times M\right) / m\left(I_{\alpha}^{(n)}\right)$. Put $I^{\infty}=\bigcap_{m} \bigcup_{n \geqq m} I^{(n)}$. Then $m\left(I^{\infty}\right)=0$ by (10). If $x \notin I^{\infty}$, then by (4) and (9) $\mu_{1, x}^{(n)}\left(J_{\alpha}^{(m)}\right)=\mu_{1, x}^{(m)}\left(J_{\alpha}^{(m)}\right)<$ $(m-2)^{-1}$ for all $\alpha \in A^{(m)}, m \geqq n_{0}(x), n \geqq m$. It follows that any cluster point $\mu_{1, x}^{\prime}$ of the sequence $\left(\mu_{1, x}^{(n)}\right)$ is a continuous measure and by the same argument as used for $\left(\mu^{(n)}\right)$, the sequence $\left(\mu_{1, x}^{(n)}\right)$ converges in the weak topology towards $\mu_{1, x}^{\prime}$. By dominated convergence, we get $\mu=\int \mu_{1, x}^{\prime} d m(x)$ and therefore $\mu_{1, x}=\mu_{1, x}^{\prime}$ a.e. The same argument works for the second coordinate.

The interiors of the rectangles $I_{\alpha}^{(n)} \times J_{\beta}^{(n)}\left(\alpha, \beta \in A^{(n)}, n \geqq 0\right)$ form clearly a basis for the topology of $I \times I$. By our construction $\mu\left(I_{\alpha}^{(n)} \times\right.$ $\left.J_{\beta}^{(n)}\right)=\mu^{(n)}\left(I_{\beta}^{(n)} \times J_{\alpha}^{(n)}\right)>0$ and therefore supp $\mu=I \times I$.

The last thing that we have to show is the extremality of $\mu$. Equivalently: if $f \in L^{\infty}(\mu)$ and the conditional expectations $E_{1} f$ and $E_{2} f$ with respect to the two coordinate projections are zero, then $f$ equals zero. Assume the contrary, i.e., $\|f\|_{\infty}=1$ and $E_{1} f=E_{2} f=0$. By the martingale convergence theorem, there exists an odd integer $n$ and $\alpha^{\prime}, \beta^{\prime} \in A^{(n)}$ such that

$$
\left|\int_{I_{\alpha^{\prime}}^{(n)} \times J_{\beta^{\prime}}^{(n)}} f d \mu\right| \geqq 2^{-1} \mu\left(I_{\alpha^{\prime}}^{(n)} \times J_{\beta^{\prime}}^{(n)}\right) .
$$

Put

$$
\begin{aligned}
B^{(n+1)} & =\left\{\left(\alpha i_{1} \cdots i_{r}, \beta i_{1} \cdots i_{s}+1 \cdots i_{r}\right): \alpha, \beta \in A^{(n)}, \varphi_{n}(\alpha, \beta)=s,\right. \\
1 & \left.\leqq i_{s}<k, 1 \leqq i_{l} \leqq k \text { for } l=1, \cdots, r, l \neq s\right\} \leqq A^{(n+1)} \times A^{(n+1)}
\end{aligned}
$$

and put 


$$
a_{\alpha \beta}=\int_{I_{\alpha}^{(n+1)} \times J_{\beta}^{(n+1)}} f d \mu \text { for } \alpha, \beta \in A^{(n+1)} .
$$

By (6) we have $\mu\left(\cup\left\{I_{\alpha}^{(n+1)} \times J_{\beta}^{(n+1)},(\alpha, \beta) \notin B^{(n+1)}\right\}\right)<c_{n} k^{-2 r} r^{-1}$. Since $\|f\|_{\infty}=1$ and $E_{1} f=E_{2} f=0$ we conclude that:

$$
\begin{gathered}
\sum_{\beta \in A^{(n+1)}}\left|\sum\left\{a_{\alpha \beta}: \alpha \in A^{(n+1)},(\alpha, \beta) \in B^{(n+1)}\right\}\right|<c_{n} k^{-2 r} 8^{-1} \\
\sum_{\alpha \in A^{(n+1)}}\left|\sum\left\{a_{\alpha \beta}: \beta \in A^{(n+1)},(\alpha, \beta) \in B^{(n+1)}\right\}\right|<c_{n} k^{-2 r} 8^{-1} .
\end{gathered}
$$

Since $2^{-1} \mu\left(I_{\alpha^{\prime}}^{(n)} \times J_{\beta^{\prime}}^{(n)}\right)-c_{n} k^{-2 r} r^{-1} \geqq 4^{-1} c_{n}$, (11) can be reformulated as follows:

$$
\begin{array}{r}
\sum\left\{a_{\alpha \beta}: I_{\alpha}^{(n \times 1)} \cong I_{\alpha^{\prime}}^{(n)}, J_{\beta}^{(n+1)} \cong J_{\beta^{\prime}}^{(n)},(\alpha, \beta) \in B^{(n+1)}\right\} \geqq 4^{-1} c_{n} \\
\text { for some } \alpha^{\prime}, \beta^{\prime} \in A^{(n)} .
\end{array}
$$

Now choose numbers $\left(a_{\alpha \beta}\right)_{(\alpha, \beta) \in B(n+1)}$ such that (12), (13), (14) are fulfilled and the cardinality of $C=\left\{(\alpha, \beta): a_{\alpha \beta} \neq 0\right\}$ becomes minimal. By (14), there exists $(\alpha, \beta) \in C$ such that $\left|a_{\alpha \beta}\right| \geqq 4^{-1} c_{n} k^{-2 r}$. Put $D_{0}=\{(\alpha, \beta)\}$ and then inductively:

$$
\begin{aligned}
D_{2 i+1} & =\left\{\left(\delta_{1}, \delta_{2}\right) \in C \backslash D_{2 i}: \exists \delta_{3} \in A^{(n+1)}:\left(\delta_{1}, \delta_{3}\right) \in D_{2 i}\right\} \\
D_{2 i} & =\left\{\left(\delta_{1}, \delta_{2}\right) \in C \backslash D_{2 i-1}: \exists \delta_{3} \in A^{(n+1)}:\left(\delta_{3}, \delta_{2}\right) \in D_{2 i-1}\right\}
\end{aligned}
$$

(e.g., $D_{2 i}$ stands for those rectangles that belong to a row determined by some rectangle from $D_{2 i-1}$, but do not belong to $D_{2 i-1}$ itself).

Now assume that $D_{i} \cap D_{j} \neq \varnothing$ for some $i<j$ and choose $i$ minimal. Assume that $i \neq 0$. Take $\left(\delta_{1}, \delta_{2}\right) \in D_{i} \cap D_{j}$. If $i$ is odd, there exists $\delta_{3} \in A^{(n+1)}$ such that $\left(\delta_{1}, \delta_{3}\right) \in D_{i-1}$. If $j$ is also odd, then $\left(\delta_{1}, \delta_{3}\right) \in D_{j} \cup D_{j-1}$ (since $\left.\left(\delta_{1}, \delta_{2}\right) \in D_{j}\right)$, but both possibilities contradict the minimality of $i$. Similarly if $j$ is even, then $\left(\delta_{1}, \delta_{3}\right) \in D_{j} \cup D_{j+1}$ which is also impossible. An analogous argument works in the case that $i$ is even. This shows that either $(\alpha, \beta) \in D_{j}$ for some $j \geqq 2$ or all $D_{j}$ are disjoint. To exclude the second possibility, observe that $\bigcup_{j \geqq 0} D_{j}$ contains with each element also the whole column corresponding to that element. Therefore, by (12):

$$
\left|\sum\left\{a_{\gamma \delta}:(\gamma, \delta) \in \bigcup_{j \geq 0} D_{j}\right\}\right|<8^{-1} c_{n} k^{-2 r} .
$$

Similarly $\bigcup_{j \geqq 1} D_{j}$ contains only complete rows, therefore by (13):

$$
\left|\sum\left\{a_{\gamma \delta}:(\gamma, \delta) \in \bigcup_{j \geqq 1} D_{j}\right\}\right|<8^{-1} c_{n} k^{-2 r} .
$$

But if the sets $D_{j}$ are disjoint, the two sums differ exactly by $a_{\alpha \beta}$ 
and this contradicts $\left|a_{\alpha, \beta}\right| \geqq 4^{-1} c_{n} k^{-2 r}$.

This shows the existence of a "loop", i.e., there exist pairwise different elements $\alpha_{1}, \cdots, \alpha_{m} \in A^{(n+1)}$, such that $\left(\alpha_{2 i-1}, \alpha_{2 i}\right),\left(\alpha_{2 i+1}, \alpha_{2 i}\right) \in$ $C$ for all $i$ (we put $\alpha_{m+1}=\alpha_{1}$ ); $m$ is clearly even. Define $a=a_{\alpha_{1} \alpha_{2}}$ and $b_{\alpha_{2_{i}-1} \alpha_{2_{i}}}=a, b_{\alpha_{2_{i}+1} \alpha_{2 i}}=-a$ for all $i$ and $b_{\alpha \beta}=0$ in the other cases. The sums in (12) and (13) are zero for $\left(b_{\alpha \beta}\right)$. If the sum in (14) would also be zero (for all choices of $\alpha^{\prime}, \beta^{\prime} \in A^{(n)}$ ), then $\left(a_{\alpha \beta}-b_{\alpha \beta}\right)$ would have the properties (12), (13), (14), contradicting our minimal choice of $\left(a_{\alpha \beta}\right)$ (since $\left.a_{\alpha_{1} \alpha_{2}}-b_{\alpha_{1} \alpha_{2}}=0\right)$. Therefore $\left(b_{\alpha \beta}\right)$ has the properties (12), (13), (14) (possibly with a smaller constant in (14)) and we will assume from now on that $a_{\alpha \beta}=b_{\alpha \beta}$ for all $\alpha, \beta$.

Now recall the definition of $A^{(n+1)}$ : we have $\alpha_{i}=\left(\rho\left(\alpha_{i}\right), \rho_{1}\left(\alpha_{i}\right)\right.$, $\left.\cdots, \rho_{r}\left(\alpha_{i}\right)\right)$ with $\rho\left(\alpha_{i}\right) \in A^{(n)}, 1 \leqq \rho_{l}\left(\alpha_{i}\right) \leqq k(l=1, \cdots, r), I_{\alpha_{i}}^{(n+1)} \leqq I_{\rho\left(\alpha_{i}\right)}^{(n)}$, $J_{\alpha_{i}}^{(n+1)} \leqq J_{\rho\left(\alpha_{i}\right)}^{(n)}$. Put $\bar{\phi}(2 i-1)=\varphi_{n}\left(\alpha_{2 i-1}, \alpha_{2 i}\right)$ and $\bar{\phi}(2 i)=\varphi_{n}\left(\alpha_{2 i+1}, \alpha_{2 i}\right)$. We may assume that $\alpha^{\prime}=\rho\left(\alpha_{1}\right), \beta^{\prime}=\rho\left(\alpha_{2}\right)$ in (14), i.e.,

$$
\sum\left\{a_{\alpha \beta}: I_{\alpha}^{(n+1)} \times J_{\beta}^{(n+1)} \cong I_{\alpha^{\prime}}^{(n)} \times J_{\beta^{\prime}}^{(n)} \neq 0 .\right.
$$

Put $t=\varphi^{-}(1)=\varphi_{n}\left(\alpha_{1}, \alpha_{2}\right)$. It follows from the definition of $B^{(n+1)}$ that for any $\alpha \in A^{(n+1)}, \gamma, \delta \in A^{(n)}$ there exists at most one $\beta \in A^{(n+1)}$ such that $(\alpha, \beta) \in B^{(n+1)}$ and $I_{\alpha}^{(n+1)} \times J_{\beta}^{(n+1)} \subset I_{\gamma}^{(n)} \times J_{\tilde{\delta}}^{(n)}$, similarly for $\alpha, \beta$ interchanged. This shows that $\varphi^{-}(l) \neq \varphi^{-}(l+1)$ for all $l$. Now put $N=\left\{l: \varphi^{-}(l)=t\right\}=\left\{l_{1}, l_{2}, l_{3}, \cdots\right\}$ with $1=l_{1}<l_{2}<l_{3} \cdots$. Since $\varphi^{-}(1)=t$, we have $\rho_{t}\left(\alpha_{1}\right)+1=\rho_{t}\left(\alpha_{2}\right)$. If $\varphi^{-}(l) \neq t$, then $\rho_{t}\left(\alpha_{l+1}\right)=\rho_{t}\left(\alpha_{l}\right)$. On the other hand, if $\varphi^{-}(l)=t$ and $l$ is even, then $\rho_{t}\left(\alpha_{l+1}\right)+1=\rho_{t}\left(\alpha_{l}\right)$ and if $l$ is odd, then $\rho_{t}\left(\alpha_{l}\right)+1=\rho_{t}\left(\alpha_{l+1}\right)$. This means that if we put $\sigma(l)=\mid\left\{j \geqq 1: l_{j}<l, l_{j}\right.$ is odd $\}|-|\left\{j \geqq 1: l_{j}<\right.$ $l, l_{j}$ is even $\} \mid$ (here $|\cdot|$ stands for the cardinality of the set), then $\rho_{t}\left(\alpha_{l}\right)=\rho_{t}\left(\alpha_{1}\right)+\sigma(l)$. In particular, for $l=m+1$, we have $\alpha_{m+1}=$ $\alpha_{1}$ and therefore $\sigma(m+1)=0$. This means that $\mid\left\{j \geqq 1: l_{j}<m+1\right.$, $l_{j}$ is odd $\}|=|\left\{j \geqq 1: l_{j}<m+1, l_{j}\right.$ is even $\} \mid$. But these numbers $l_{j}$ determine those indices $(\alpha, \beta)$ for which $a_{\alpha \beta} \neq 0$ and $I_{\alpha}^{(n+1)} \times J_{\beta}^{(n+1)} \cong$ $I_{\alpha^{\prime}}^{(n)} \times J_{\beta^{\prime}}^{(n)}$. If $l_{j}$ is odd, then $a_{\alpha \beta}=+a$ and if it is even then $a_{\alpha \beta}=$ -a. By summing up we would get: $\sum\left\{a_{\alpha \beta}: I_{\alpha}^{(n+1)} \times J_{\beta}^{(n+1)} \cong I_{\alpha^{\prime}}^{(n)} \times J_{\beta^{\prime}}^{(n)}\right\}=$ 0 , contrary to (14). This contradiction proves that $\mu$ is extremal.

Finally since all the measures in the two disintegrations of $\mu$ are continuous, it follows that any graph or inverse graph has $\mu$ measure zero.

\section{REFERENCES}

1. J. R. Brown, Approximation theorems for Markov operators, Pacific J. Math., 16 (1966), 13-23.

2. J. R. Brown and R. C. Shiflett, On extreme double stochastic measures, Michigan Math. J., 17 (1970), 249-254. 
3. A. Diego and A. Germani, Extremal measures with prescribed marginals (finite case), J. Combinatorial Theory, 13 (1972), 353-366.

4. R. G. Douglas, On extremal measures and subspace density, Michigan Math. J., 11 (1964), 243-246.

5. - On extremal measures and subspace density II, Proc. Amer. Math. Soc., 17 (1966), 1363-1365.

6. N. Dunford and J. T. Schwartz, Linear Operators I, Interscience Publishers, New York-London, 1958.

7. J. Lindenstrauss, A remark on extreme doubly stochastic measures, Amer. Math. Monthly, 72 (1965), 379-382.

8. L. Mirsky, Results and problems in the theory of doubly stochastic matrices, Z. Wahrscheinlichkeitstheorie verw. Gebiete, 1 (1963), 319-334.

9. K. P. Parthasarathy, Probability Measures on Metric Spaces, Academic Press, New York, 1967.

10. J. V. Ryff, Orbits of $L^{1}$-functions under doubly stochastic transformations, Trans. Amer. Math. Soc., 117 (1965), 92-100.

11. H. H. Schaefer, Topological Vector Spaces, Springer-Verlag, Berlin-Heidelberg-New York 1971.

[12] T. L. Seethoff and R. C. Shiflett, Doubly stochastic measures with prescribed support, Z. Wahrscheinlichkeitstheorie verw. Gebiete, 41 (1977), 283-288.

13. V. N. Sudakov, Geometric problems in the theory of infinite dimensional probability distributions (in Russian), Trudy Mat. Inst. Steklov, 141 (1976).

Received January 13, 1981 and in revised form May 21, 1981.

UNIVERSITÄT WIEN

STRUDLHOFGASSE 4

A-1090 WiEn, Austria 



\title{
PACIFIC JOURNAL OF MATHEMATICS
}

\section{EDITORS}

\author{
DONALD BABBITT (Managing Editor) \\ University of California \\ Los Angeles, CA 90024 \\ Hugo RossI \\ University of Utah \\ Salt Lake City, UT 84112 \\ C. C. MOORE and Arthur Agus \\ University of California \\ Berkeley, CA 94720
}

\section{J. DugundjI}

Department of Mathematics

University of Southern California

Los Angeles, CA 90007

R. FinN and J. Milgram

Stanford University

Stanford, CA 94305

\section{ASSOCIATE EDITORS}
R. ARENS
E. F. BeCKENBACH
B. H. NeumanN
F. WOLF
K. YoshidA

\section{SUPPORTING INSTITUTIONS}

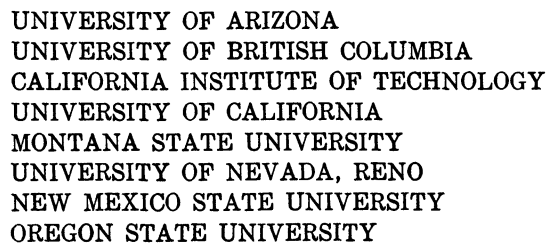

UNIVERSITY OF ARIZONA

UNIVERSITY OF BRITISH COLUMBIA

CALIFORNIA INSTITUTE OF TECHNOLOGY

UNIVERSITY OF CALIFORNIA

MONTANA STATE UNIVERSITY

UNIVERSITY OF NEVADA, RENO

NEW MEXICO STATE UNIVERSITY

OREGON STATE UNIVERSITY

\author{
UNIVERSITY OF OREGON \\ UNIVERSITY OF SOUTHERN CALIFORNIA \\ STANFORD UNIVERSITY \\ UNIVERSITY OF HAWAII \\ UNIVERSITY OF TOKYO \\ UNIVERSITY OF UTAH \\ WASHINGTON STATE UNIVERSITY \\ UNIVERSITY OF WASHINGTON
}

The Supporting Institutions listed above contribute to the cost of publication of this Journal, but they are not owners or publishers and have no responsibility for its content or policies.

Mathematical papers intended for publication in the Pacific Journal of Mathematics should be in typed form or offset-reproduced, (not dittoed), double spaced with large margins. Please do not use built up fractions in the text of the manuscript. However, you may use them in the displayed equations. Underline Greek letters in red, German in green, and script in blue. The first paragraph or two must be capable of being used separately as a synopsis of the entire paper. Please propose a heading for the odd numbered pages of less than 35 characters. Manuscripts, in triplicate, may be sent to any one of the editors. Please classify according to the scheme of Math. Reviews, Index to Vol. 39. Supply name and address of author to whom proofs should be sent. All other communications should be addressed to the managing editor, or Elaine Barth, University of California, Los Angeles, California, 90024.

50 reprints to each author are provided free for each article, only if page charges have been substantially paid. Additional copies may be obtained at cost in multiples of 50 .

The Pacific Journal of Mathematics is issued monthly as of January 1966. Regular subscription rate: $\$ 102.00$ a year (6 Vols., 12 issues). Special rate: $\$ 51.00$ a year to individual members of supporting institutions.

Subscriptions, orders for numbers issued in the last three calendar years, and changes of address shoud be sent to Pacific Journal of Mathematics, P.O. Box 969, Carmel Valley, CA 93924, U.S.A. Old back numbers obtainable from Kraus Periodicals Co., Route 100, Millwood, NY 10546.

\section{PUBLISHED BY PACIFIC JOURNAL OF MATHEMATICS, A NON-PROFIT CORPORATION}

Printed at Kokusai Bunken Insatsusha (International Academic Printing Co., Ltd.). 8-8, 3-chome, Takadanobaba, Shinjuku-ku, Tokyo 160, Japan. 


\section{Pacific Journal of Mathematics}

Vol. 99, No. $2 \quad$ June, 1982

Thomas E. Armstrong and Karel Libor Prikry, On the semimetric on a

Boolean algebra induced by a finitely additive probability measure . . . .249

Walter Russell Bloom, Strict local inclusion results between spaces of

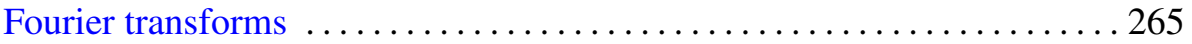

Richard Clark Brown, Notes on generalized boundary value problems in

Banach spaces. II. Infinite-dimensional extension theory ........... 271

Sui Sun Cheng, Isoperimetric eigenvalue problem of even order differential

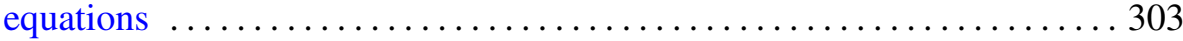

Lung O. Chung and Jiang Luh, Derivations of higher order and

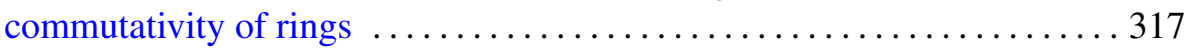

Ali Ahmad Fora, A fixed point theorem for product spaces . . . . . . . . . 327

Barry J. Gardner, Radical classes of regular rings with Artinian primitive

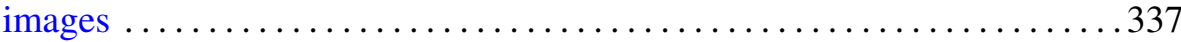

John Brady Garnett and Peter Wilcox Jones, BMO from dyadic BMO . . . 351

Allen E. Hatcher, On the boundary curves of incompressible surfaces . . . . 373

Richard Howard Hudson and Kenneth S. Williams, Resolution of

ambiguities in the evaluation of cubic and quartic Jacobsthal sums .....379

Viktor Losert, Counter-examples to some conjectures about doubly

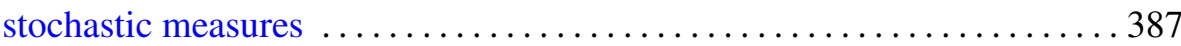

Kenneth Derwood Magill, Jr., P. R. Misra and Udai Bhan Tewari,

Structure spaces for sandwich semigroups

Mark Mandelker, Continuity of monotone functions

Kenneth Guy Miller, An index theorem and hypoellipticity on nilpotent Lie groups ......................................... 419

Evelyn M. Nelson, Homomorphisms of mono-unary algebras . . . . . . . . . 427

Marvin E. Ortel, The support of an extremal dilatation . . .

R. S. Pathak and O. P. Singh, Finite Hankel transforms of distributions . . . 439

Richard Cole Penney, The theory of ad-associative Lie algebras

Linda Ruth Sons, Zero distribution of functions with slow or moderate

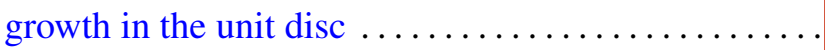

Russell Bruce Walker, Transversals to laminations 